\title{
NUEVOS MATERIALES DE TITANOSAURIOS (SAUROPODA) EN EL CRETÁCICO SUPERIOR DE MATO GROSSO, BRASIL
}

\author{
ALDIRENE COSTA FRANCO-ROSAS \\ Departamento de Geologia, UFRJ, Cidade Universitária, Ilha do Fundão, 21940-940, Rio de Janeiro, RJ, Brasil. \\ aldirene@terra.com.br \\ LEONARDO SALGADO \\ Museo de Geología y Paleontología, Universidad Nacional del Comahue, Buenos Aires 1400, 8300 Neuquén, Argentina. \\ lsalgado@uncoma.edu.ar \\ CLAUDIO FABIÁN ROSAS \\ Departamento de Geologia Aplicada, IGCE, UNESP, Uruguai, 296, 13420.514, Piracicaba, SP. Brasil. rosascf@terra.com.br \\ ISMAR DE SOUZA CARVALHO \\ Departamento de Geologia, UFRJ, Cidade Universitária, Ilha do Fundão, 21940-940, Rio de Janeiro, RJ, Brasil. \\ ismar@geologia.ufrj.br
}

\begin{abstract}
RESUMO - São descritos restos ósseos provenientes da bacia Bauru (Formação Cambambe, Mato Grosso), atribuídos a Gondwanatitan, e definido um novo taxon de titanossauros: Aeolosaurini. A presença deste clado em níveis cronocorrelatos estratigraficamente na Bacia Bauru (estados de São Paulo, Minas Gerais e Mato Grosso) e em algumas localidades do Norte da Patagônia (Argentina) sugere a existência de uma província paleobiogeográfica para estes animais e não indicando, necessariamente, a livre circulação dos saurópodos entre a região brasileira e Argentina durante o Cretáceo Superior.
\end{abstract}

Palavras-chave: Titanosauria, Gondwanatitan, Cretáceo Superior, Mato Grosso.

ABSTRACT - NEW MATERIALS OF TITANOSAURS (SAUROPODA) FROM THE UPPER CRETACEOUS OF MATO GROSSO STATE, BRAZIL. - New material from Bauru Basin (Cambambe Formation, Mato Grosso State), assigned to Gondwanatitan, is described, and a new taxon of titanosaurs, Aeolosaurini, is defined. The presence of this group in cronocorrelated stratigraphic levels of Bauru Basin (throughout São Paulo, Minas Gerais and Mato Grosso states) and in many localities of North Patagonia may suggest the existence of a wide paleobiogeographical province; but it does not necessarily favor the hypothesis of the free-crossing of the sauropods through an hypothetical geographical barrier that would have isolated most of the Bauru fauna in southeastern of Brazil.

Key words: Titanosauria, Gondwanatitan, Upper Cretaceous, Mato Grosso.

\section{INTRODUCCIÓN}

El estudio de los saurópodos titanosaurios es de gran importancia en el ámbito de la paleontología de vertebrados, en parte debido a la posibilidad de correlacionar las unidades geológicas portadoras de sus restos. Con excepción de la Antártida, los titanosaurios se registran en niveles del Cretácico Superior de todos los continentes (Salgado et al., 1997b). Sólo en América del Sur, en dónde se documentan con mayor abundancia, se han reconocido al menos 11 géneros de titanosaurios.

En Brasil, el hallazgo de restos de titanosaurios se ha incrementado notablemente en los últimos años. Actualmen- te, el registro se concentra en dos áreas principales: la Cuenca de São Luís (Albiano-Cenomaniano) (Medeiros \& Schultz, 2000) y, sobre todo, la Cuenca de Bauru (Cretácico Superior) (Arid \& Vizotto, 1971; Mezzalira, 1989; Bertini et al., 1993, 2000; Kellner \& Azevedo, 1999; Santucci \& Bertini, 2001, 2002), de donde provienen los materiales que presentamos en esta contribución. Los mismos, los primeros elementos óseos de titanosaurios provenientes del Estado de Mato Grosso, fueron extraídos en 2001 de una nueva localidad fosilífera, ubicada en Morro do Cambambe.

Hasta el momento, algunos de los elementos hallados en la cuenca de Baurú (en los Estados de São Paulo y Minas Gerais) han sido asignados o vinculados a géneros 
reconocidos en Patagonia (Salgado et al., 1997a,b). Esa supuesta relación, sumada a otras evidencias, ha dado pie a hipótesis paleobiogeográficas que comprende al conjunto de la fauna de vertebrados continentales de la Cuenca de Bauru (Bertini et al., 1993). En esta contribución, discutimos las posibles relaciones filogenéticas del saurópodo de Mato Grosso, y su aporte al conocimiento de la paleobiogeografía del Centro-Sur de Brasil durante el Cretácico Superior.

Abreviaturas institucionales. MPMA, Museu de Paleontologia, Centro Cultural, Prefeitura Municipal de Monte Alto, Monte Alto; CPP, Centro de Pesquisas Paleontológicas "Llewellyn Ivor Price", Distrito de Petrópolis, Uberaba, MG; MP, Museu de Paleontologia da Universidade Federal do Mato Grosso; Brasil; MN, Museu Nacional/Universidade Federal do Rio de Janeiro, Rio de Janeiro; MPCA, Museo Provincial "Carlos Ameghino", Cipolletti, Río Negro; MJGRN, Museo Jorge Gerold, Ingeniero Jacobacci, Río Negro; MACN-RN, Museo Argentino de Ciencias Naturales "Bernardino Rivadavia", colección Río Negro, Buenos Aires.

\section{GEOLOGÍA REGIONAL}

La Cuenca Bauru es una depresión post-Gondwánica desarrollada en el centro sur de la plataforma sudamericana por subsidencia termo-mecánica en el Cretácico Superior. En esta área de aproximadamente $370.000 \mathrm{~km}^{2}$ se depositó una secuencia sedimentaria predominantemente arenosa con espesores máximos conservados de trescientos metros (Fúlfaro \& Perinotto, 1996; Fernandes \& Coimbra, 1996). Esta cuenca se divide en los grupos Caiuá y Bauru.

El Grupo Bauru representa una importante unidad Cretácica continental brasileña siendo el blanco de numerosas investigaciones geológicas y paleontológicas en las últimas décadas, especialmente en los estados de São Paulo y Minas Gerais.

Bauer \& Largher (1958) fueron los primeros autores en mencionar rocas equivalentes del Grupo Bauru en el Estado de Mato Grosso, en estudios realizados en las proximidades del Rio Roncador. Esta unidad geológica aparece al norte y nordeste de la ciudad de Chapada dos Guimarães donde se localiza el Morro de Cambambe (Figuras 1 y 2).

Weska et al. (1988) mapeando la región de Poxoréu propusieron la formalización del Grupo Bauru en el Estado do Mato Grosso. Posteriormente Weska (1996) y Weska et al. (1996) sintetizaron las informaciones de la región de Chapada dos Guimarães, definiendo las siguientes formaciones como pertenecientes al Grupo Bauru (de la base hacia el techo): formaciones Paredão Grande, Quilombinho, Cachoeira do Bom Jardim y Cambambe.

La Formación Paredão Grande está constituida por un conjunto de rocas volcánicas cuyos derrames basálticos aparecen en las regiones de Chapada dos Guimarães, Poxoréu y Don Aquino (Weska, 1996; Zolinger, 2000) y están datadas en 83,9 $\pm 0,4$ Ma (Gibson et al., 1997). Estos derrames se encuentran



Figura 1. Ubicación geográfica del Estado de Mato Grosso y de la ciudad de Chapada dos Guimarães, Brasil

Figure 1. Geographical location of the Mato Grosso State and the Chapada dos Guimaraes city, Brazil.

interdigitados con las formaciones Quilombinho y Cachoeira do Bom Jardim.

Según Weska (1996), la Formación Cambambe está compuesta por areniscas y areniscas arcillosas microconglomerádicas a conglomerádicas con lentes de conglomerados a microconglomerados, brechas intraformacionales, areniscas de granulometría fina, media y microconglomerádica, silcretes y raras limonitas. En la región de Morro do Cambambe el espesor de la formación puede llegar a 300 m (Weska, 1996). Según Roxo (1937), la edad de la Formación Cambambe es Cretácico Superior, por el registro en la Chapada dos Guimarães de fósiles de Crocodylia, dinosaurios y Chelonia.

El área de procedencia del material que describimos en este trabajo se sitúa en las laderas del Cerro Cambambe a $40 \mathrm{~km}$ al norte de la ciudad de Chapada dos Guimarães, Mato Grosso. Se trata de areniscas, areniscas limosas y areniscas arcillosas con nódulos calcáreos. Todo el material se encuentra fuertemente alterado y soportando el desarrollo de un suelo con acumulación, tanto de materia orgánica como de material arcilloso. Las areniscas son de granulometría media a fina de color rojo, de base neta con continuidad lateral definiendo cuerpos tabulares de $0,30 \mathrm{a}$ $2 \mathrm{~m}$ de espesor. Por encima se encuentran areniscas granodecrecientes, blancas, de base neta y erosiva con intraclastos arcillosos. No se observan estructuras sedimentarias pero sí una variación litológica lateral, disminuyendo el porcentaje de arenisca y aumentando el de arcilla y precipitados químicos. Estos cuerpos poseen una sección de tipo canal con espesores máximos de 0,5 a $1,5 \mathrm{~m}$. Posteriormente existe una capa de suelo de aproxi- 

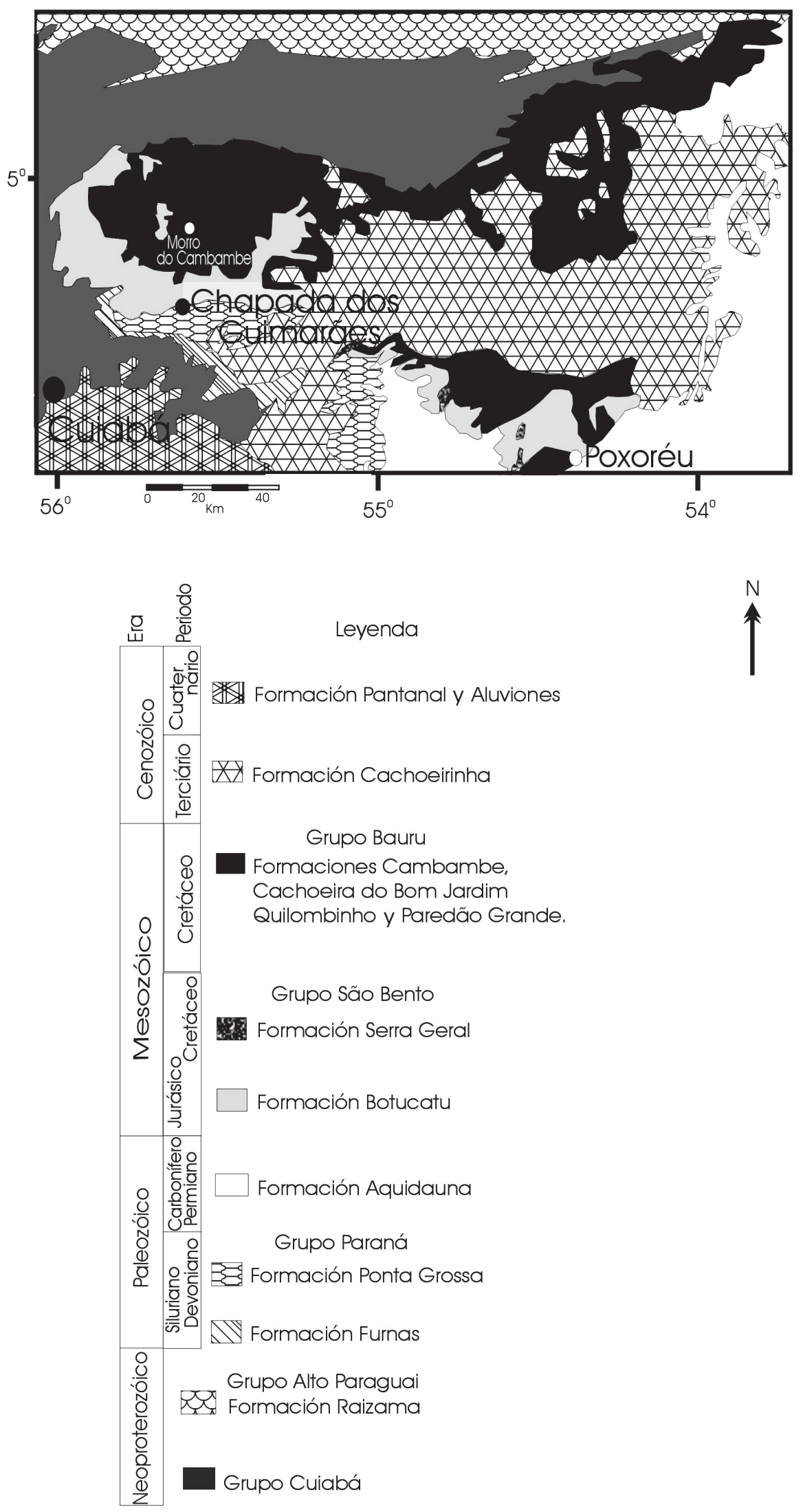

Figura 2. Mapa geológico simplificado de la localidad de colección.

Figure 2. Simplified geological map of the collecting site. 
madamente $0,4 \mathrm{~m}$ de espesor (Figura 3 ).

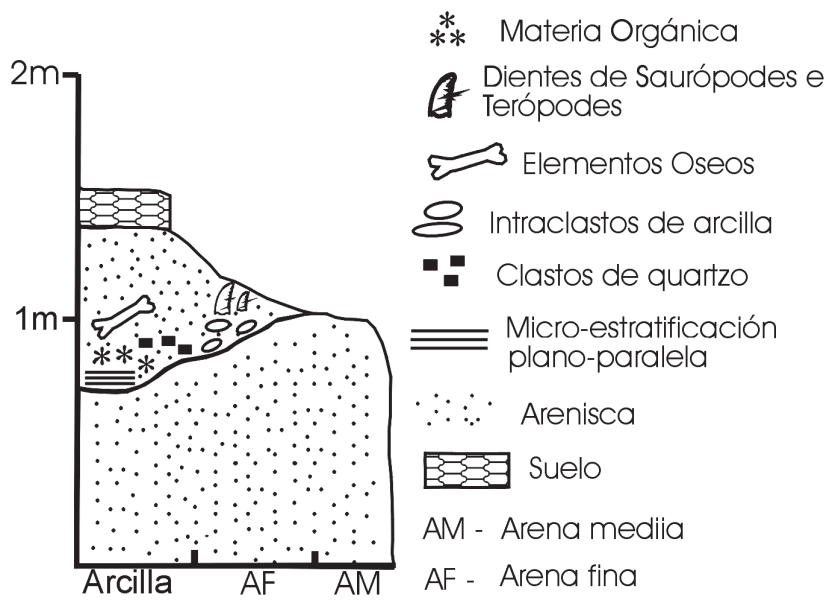

Figura 3. Perfil estratigráfico esquemático del afloramiento. Figure 3. Schematic stratigraphic section of the outcrop.

\section{PALEONTOLOGÍA SISTEMÁTICA}

\author{
SAUROPODA Marsh, 1878 \\ NEOSAUROPODA Bonaparte, 1986 \\ TITANOSAURIFORMES Salgado et al., 1997 a \\ TITANOSAURIA Bonaparte \& Coria, 1993 \\ SALTASAURINAE Powell, 1992 \\ AEOLOSAURINI nuevo taxón
}

Definición. El clado más amplio que comprende a Aeolosaurus rionegrinus y Gondwanatitan faustoi pero no a Saltasaurus loricatus ni a Opisthocoelicaudia skarzynskii (Figura 4).



Figura 4. Hipótesis sobre las relaciones filogenéticas de los Titanosauria, indicando la posición sugerida de los Aelosaurin (modificado de Salgado et al., 1997a,b).

Figure 4. Hypothetical phylogenetic relationships of the Titanosauria, showing the suggested placement of the Aelosaurini (modified from Salgado et al., 1997a,b).
Diagnosis. Los Aeolosaurini se diferencian de los restantes titanosaurios por poseer: cuerpos caudales anteriores y medios con el margen anterior inclinado hacia delante; arcos neurales dispuestos sobre el borde anterior del centro en las vértebras caudales medias; espina neural inclinada hacia adelante, al menos en las caudales medias; prezigapófisis alargadas en las caudales anteriores y medias, en correlación con el desplazamiento del arco neural hacia delante; facetas articulares de las prezigapófisis y postzigapófisis alargadas anteroposteriormente, al menos en las caudales anteriores y medias.

Especies incluidas. Aeolosaurus rionegrinus, Gondwanatitan faustoi y Rinconsaurus caudamirus.

Materiales referidos a Aeolosaurini. Aeolosaurus sp. MPMA/sin número (Santucci \& Bertini, 2001); Aeolosaurus sp. CPP 298 (Santucci \& Bertini, 2001); Aelosauros sp. MPCA 27100 (Salgado et al., 1997b) y MPAC 27174 (Salgado \& Coria, 1983); Aelosauros rionegrinus? MACN-RN 107 (Powell, 1987).

Gondwanatitan sp. indet.

Material. MP 285, centro caudal anterior incompleto (Figura 5); MP 288, arco neural (Figura 6); MP 287, vértebra caudal media incompleta (Figura 7); MP 284, vértebra caudal posterior completa (Figura 8); MP 286, tibia izquierda incompleta (Figura 9).

\section{Descripción}

Vértebras Caudales. El cuerpo vertebral anterior (MP 285) es voluminoso, y la cara articular posterior es levemente. convexa. La parte anterior del centro vertebral está rota, de modo que no es posible establecer si se trata de un cuerpo procélico o biconvexo, como ocurre en la primera vértebra caudal de algunos titanosaurios.

El arco neural incompleto (MP 288) ha preservado la base de la espina neural y las bases de ambas prezigapófisis. Las postzigapófisis son alargadas anteroposteriormente. $\mathrm{La}$ vértebra caudal media (MP 287) no ha preservado la espina neural ni los extremos de las prezigapófisis. El cuerpo vertebral es tan alto como ancho. Las caras laterales del centro vertebral son cóncavas, tanto en sentido anteroposterior como dorsoventral y la cara ventral es estrecha. En vista lateral, el borde anterior del centro vertebral se inclina claramente hacia adelante y la articulación posterior es fuertemente procélica, como en la mayoría de los titanosaurios. El arco neural se halla desplazado hacia el borde anterior del centro vertebral, en tanto que las prezigapófisis se dirigen hacia adelante y levemente hacia arriba. La vértebra caudal posterior (MP 284) posee el centro vertebral relativamente bajo. En vista lateral el centro vertebral es fuertemente cóncavo en su margen ventral. La articulación posterior es prominente. El arco neural está dispuesto sobre la mitad anterior del centro y la espina neural se halla inclinada posteriormente. 

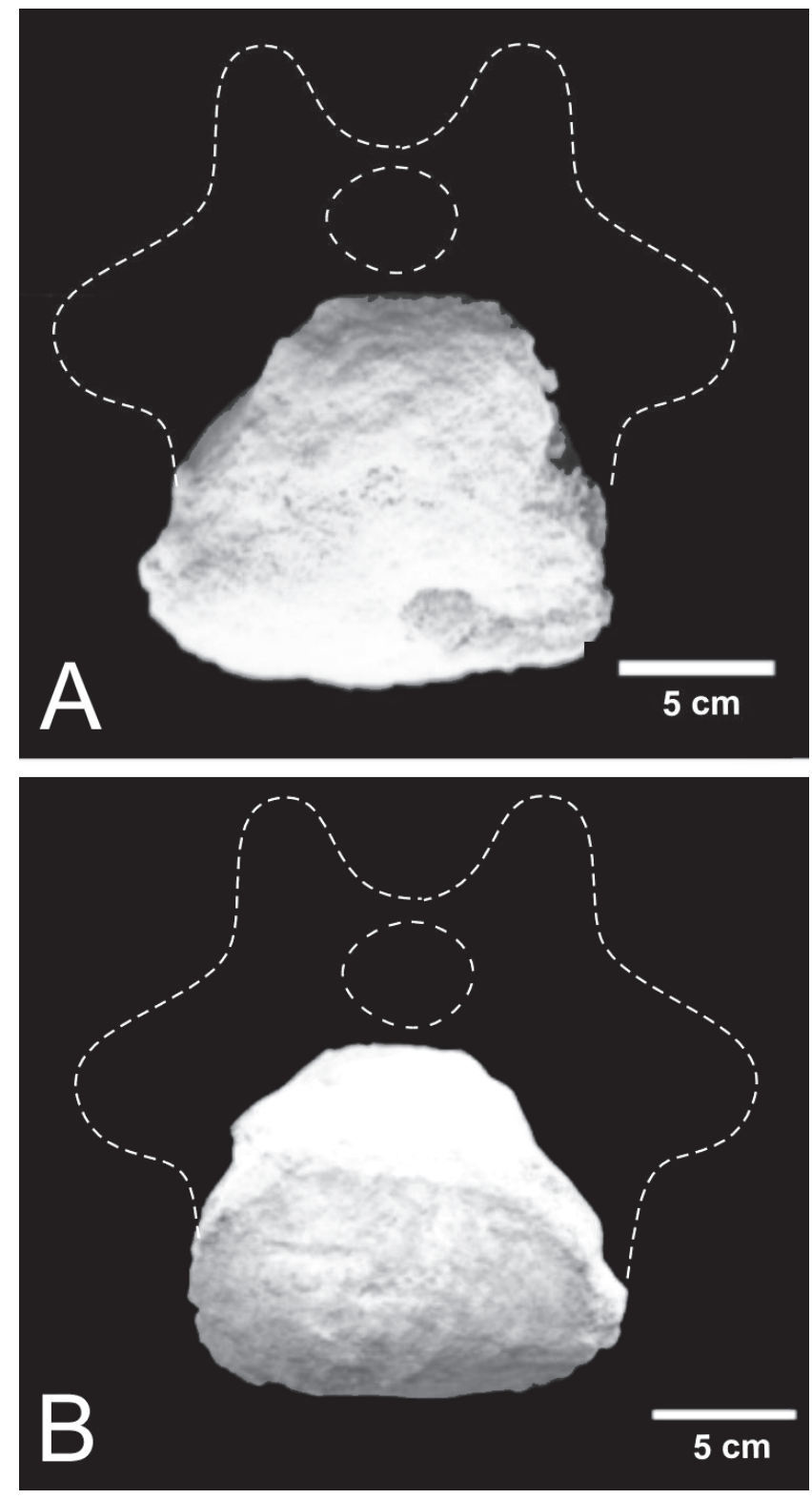

Figura 5. Gondwanatitan sp., MP 285: centro caudal anterior en vista antero-ventral (A) y vista postero-dorsal (B).

Figure 5. Gondwanatitan sp., MP 285: anterior caudal centrum in antero-ventral (A) and postero-dorsal views (B).

Tibia. Se ha preservado una tibia izquierda (MP 286) casi completa (Figura 9); sólo faltan su extremo distal y la región anterior del extremo proximal, aunque aparentemente este sector no se encontraba tan elevado como en Gondwanatitan faustoi (Kellner \& Azevedo, 1999, fig. 21). En líneas generales, es una pieza esbelta, algo más delgada que la de Aeolosaurus (Powell, 1986).

\section{DISCUSIÓN}

Los materiales que hemos presentado en este trabajo presentan un conjunto de características que permiten asignarlos a los Aeolosaurini nuevo táxon. Entre estas se
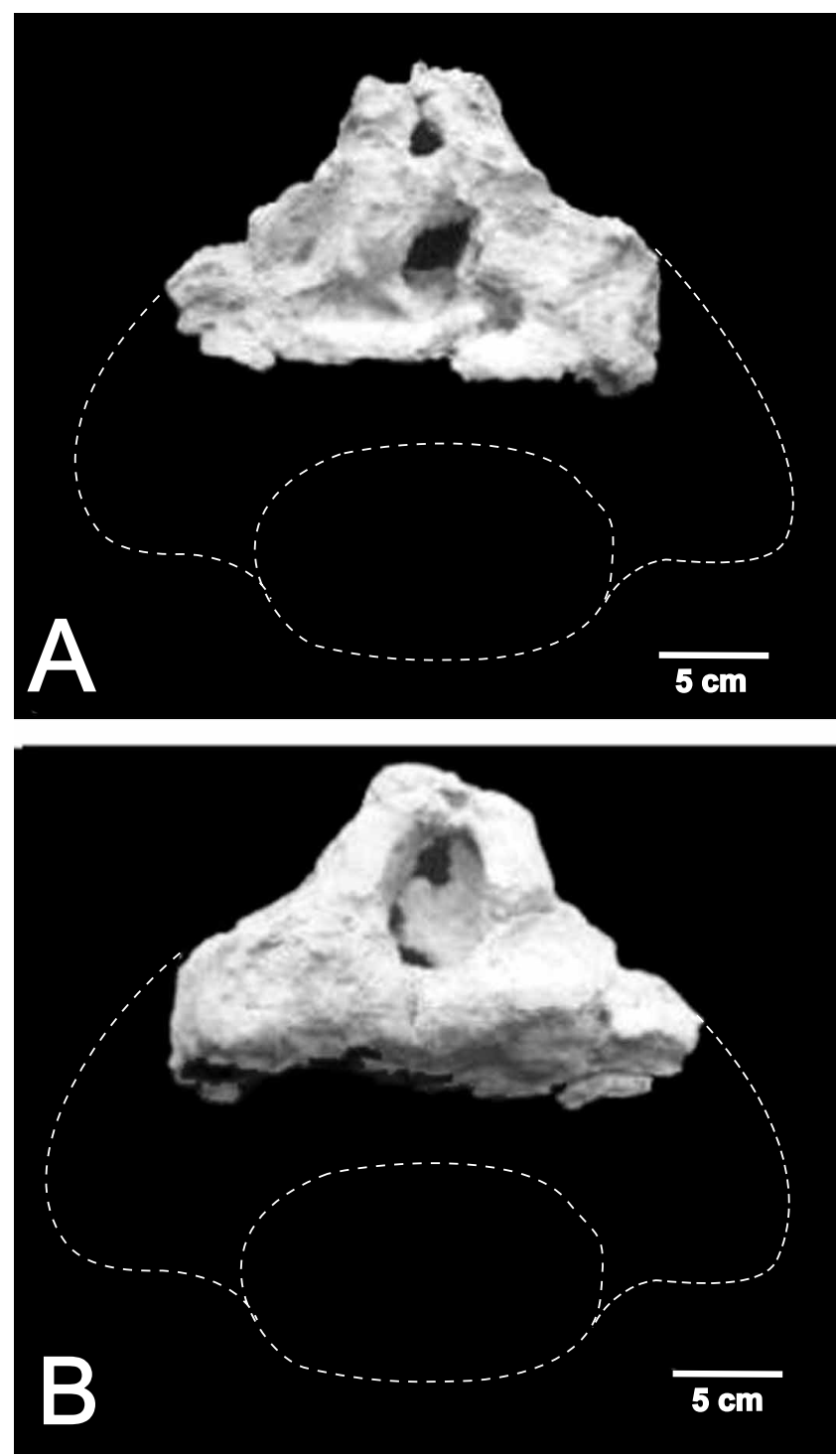

Figura 6. Gondwanatitan sp., MP 288: arco neural caudal anterior en vista anterior (A) y vista posterior (B).

Figure 6. Gondwanatitan sp., MP 288: anterior caudal neural arch in anterior (A) and posterior views (B).

encuentran: el arco neural desplazado hacia el borde anterior del centro vertebral en las caudales medias; el margen anterior del centro vertebral en las caudales medias inclinado hacia delante y el eje mayor de las postzigapófisis orientado anteroposteriormente. Si bien los integrantes de este nuevo clado de saurópodos pueden identificarse principalmente a partir de características de las vértebras caudales, consideramos que la totalidad de los materiales registrados en la localidad de Morro do Cambambe pertenece a este nuevo grupo. Posiblemente, los restos hallados correspondan incluso a una misma especie de titanosaurio, ya que los mismos se encontraron asociados en un mismo nivel estratigráfico. En particular, la tibia (MP 286) es comparable a la de Gondwanatitan faustoi (Kellner \& Azevedo, 1999, fig. 21), el único aeolosaurino brasileño conocido a partir de su 

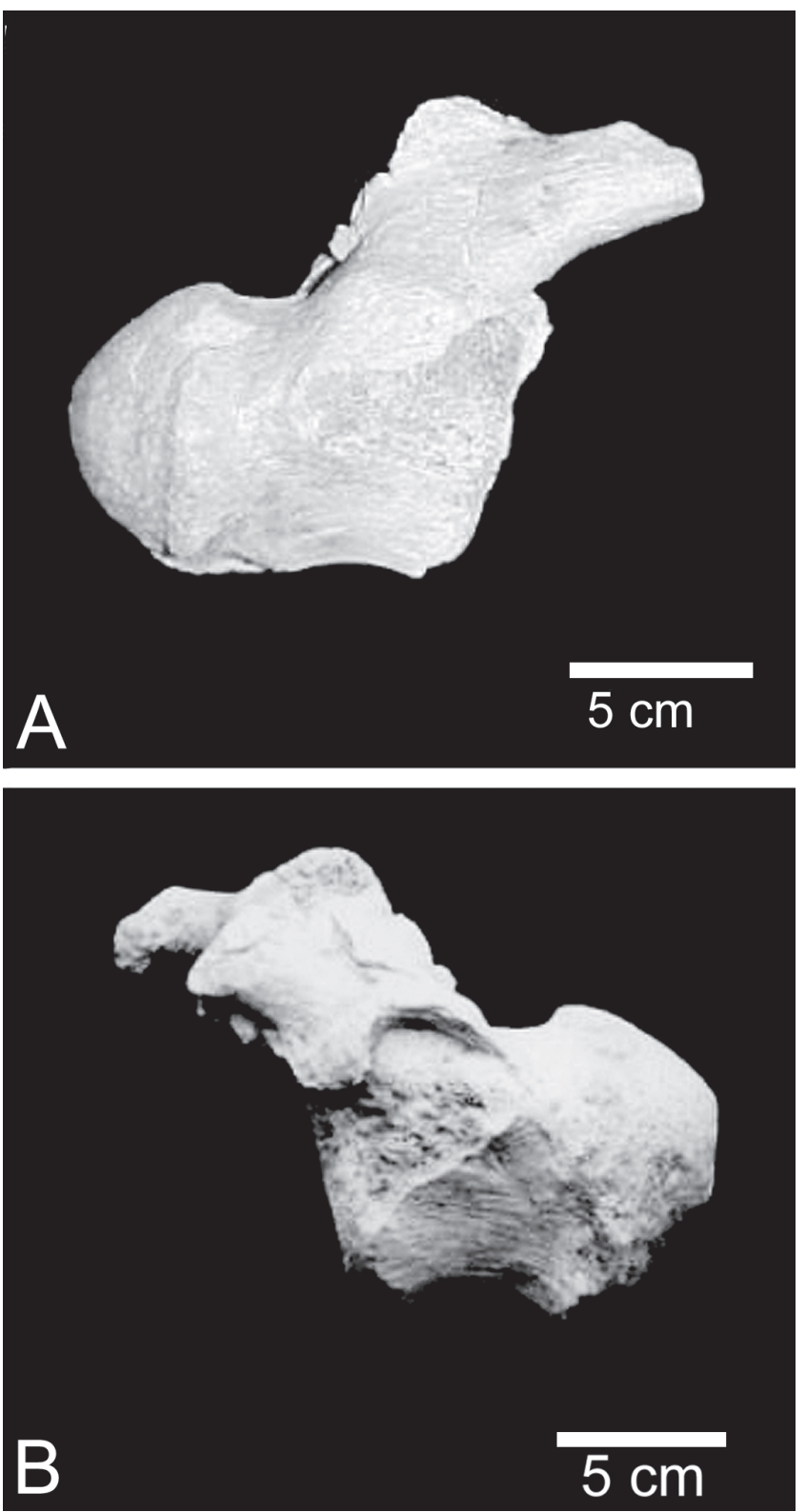

Figura 7. Gondwanatitan sp. MP 287: vértebra caudal media en vista lateral derecha (A) y lateral izquierda (B).

Figure 7. Gondwanatitan sp. MP 287: mid caudal vertebra in lateral right $(\mathbf{A})$ and lateral left $(\mathbf{B})$ views.

esqueleto apendicular (escápula, húmero, ileon, isquion, pubis, y tibia). La tibia del holotipo de Aeolosaurus rionegrinus es algo más robusta que la de Gondwanatitan, al igual que el resto de los huesos apendiculares. Los otros elementos hallados en Morro do Cambambe, en especial las vértebras caudales, también guardan similitud, tanto en su morfología como en su reducido tamaño, con la especie de Álvares Machado (Gondwanatitan faustoi, Estado de São Paulo). Es por esta razón que referimos tentativamente el conjunto de materiales al género Gondwanatitan.

Aparentemente, los Aeolosaurini se encuentran restringidos al Cretácico Superior de Sudamérica, por lo que es muy
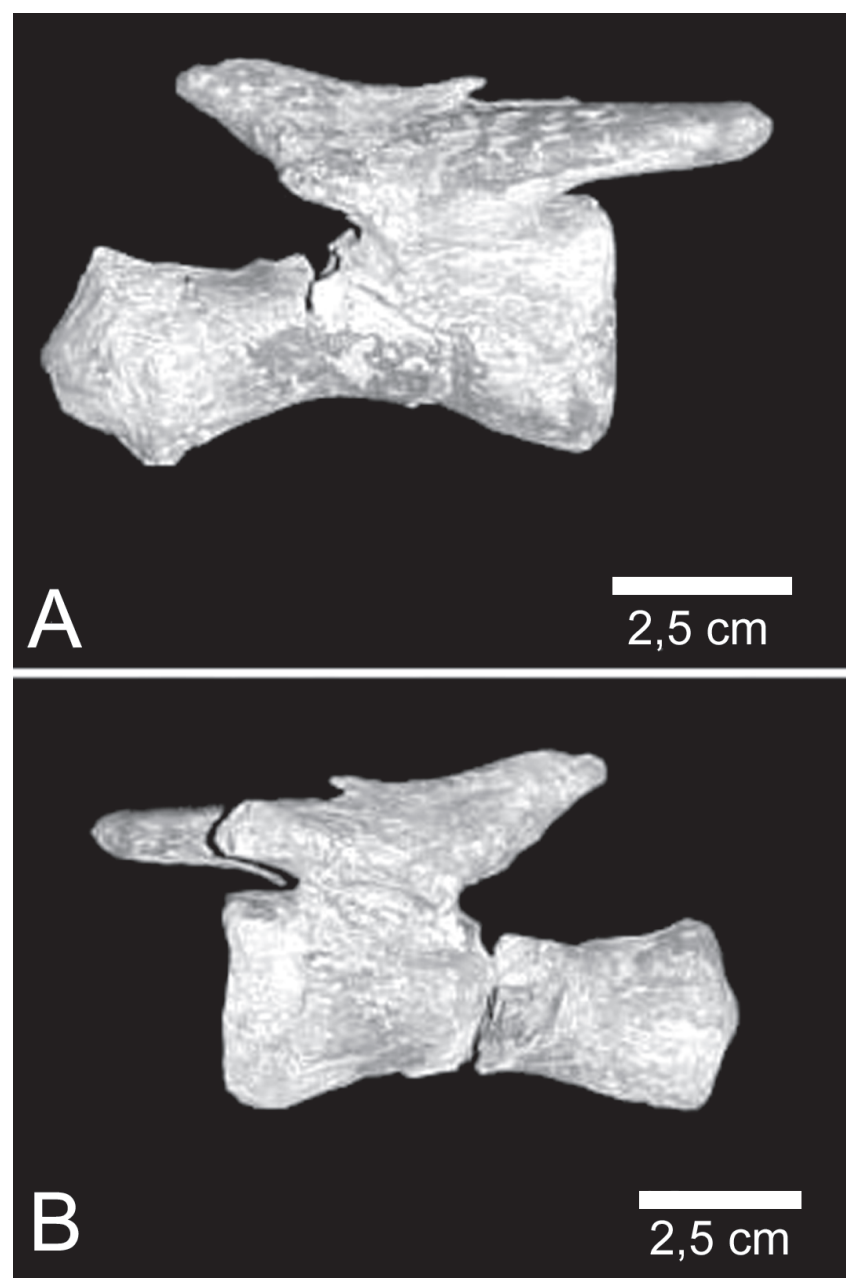

Figura 8. Gondwanatitan sp. MP 284: vértebra caudal posterior en vista lateral derecha $(\mathbf{A})$ y lateral izquierda (B).

Figure 8. Gondwanatitan sp. MP 284: posterior caudal vertebra in lateral right $(\mathbf{A})$ and lateral left $(\mathbf{B})$ views.

posible que su diferenciación evolutiva haya ocurrido luego de la apertura del Atlántico Sur (Cretácico Inferior, Albiano). Por otra parte, la ausencia de Aeolosaurini en otros puntos de Gondwana, como Madagascar y la India, sugiere la existencia, en el Cretácico Superior, de una barrera geográfica que habría impedido la dispersión de ese grupo hacia esos continentes. Ciertas evidencias paleontológicas, como los restos de testudines y cocodrilomorfos, parecen indicar un cierto endemismo de la fauna del Grupo Bauru. Para Santucci y Bertini (2001), el registro de Aeolosaurus en los estados de São Paulo y Minas Gerais en niveles correspondientes al Grupo Bauru, y en diferentes localidades del Norte de Patagonia, indicaría que la presunta barrera geográfica establecida en los bordes de la Cuenca Bauru habría permitido el libre pasaje de los grandes saurópodos. Sin embargo, para resolver sobre esta cuestión hay que determinar, en primer término, las edades mínimas de diferenciación de los diferentes grupos involucrados (e.g. Aeolosaurus, Gondwanatitan, Aeolosaurini, etc.) y contrastarlas con la edad del establecimiento de la presunta barrera geográfica. 


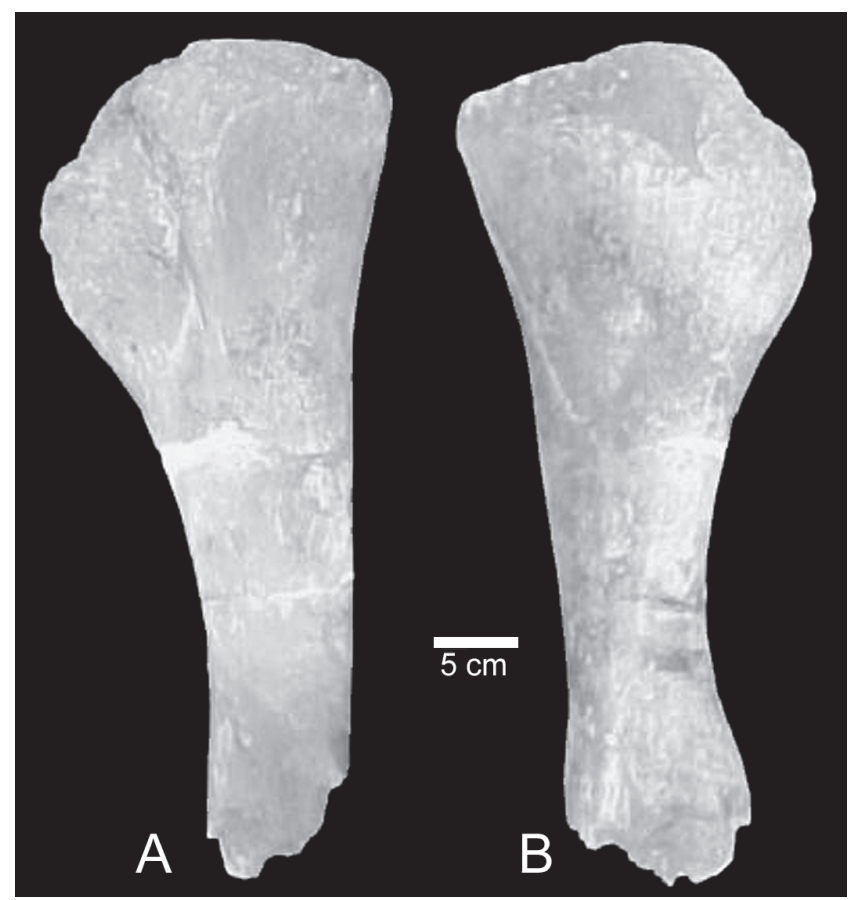

Figura 9. Gondwanatitan sp., MP 286: tibia izquierda en vistas lateral (A) y medial (B).

Figure 9. Gondwanatitan sp., MP 286: left tibia in lateral (A) and medial views (B).

En este sentido, la presencia de aeolosaurinos a uno y otro lado de la hipotética barrera de aislamiento puede simplemente deberse a que el tiempo dediferenciación del grupo (sea Aeolosaurus o Aeolosaurini) es anterior al establecimiento de la presunta barrera, en tanto que los demás grupos (subgrupos de testudines y crocodilomorfos), pudieron hacerlo después.

Desconocemos si los Aeolosaurini presentes en las formaciones del Grupo Bauru poseen más relación entre sí que con alguna forma patagónica. Por lo tanto, no es posible sostener que el registro de estos saurópodos dentro y fuera de los límites de la Cuenca es una evidencia a favor de la permeabilidad de la barrera para estos grandes dinosaurios.

\section{AGRADECIMIENTOS}

Agradecemos a la Fundação Carlos Chagas Filho de Amparo à Pesquisa do Estado de Río de Janeiro (FAPERJ) y al CNPQ (Proc. $\mathrm{n}^{\circ}$ 300571/03-8) por el financiamiento en los trabajos de campo y por la beca de doctorado (ACFR). Al Álvaro Pizzato Cuadros (UFMT) por la viabilización del trabajo, por el apoyo logístico, por las informaciones geológicas y paleontológicas. A Ricardo Weska (UFMT) por las indicaciones bibliográficas y valiosos esclarecimientos en la geología regional y local. A Rubens.; José, Santo y sus esposas;. Neide, y Edson que posibilitaron el acceso al afloramiento. Al los compañeros de campaña: Leonardo P. Marconato, Claudia M. M. Ribeiro, Paulo F. Souto y Francisco J. C. Martins.

\section{REFERENCIAS}

Arid, F.M. \& Vizotto, L.D. 1971. Antarctosaurus brasiliensis, um novo saurópodo do Cretáceo Superior do sul do Brasil. In: CONGRESSO BRASILEIRO DE GEOLOGIA, 25, 1971. Anais, São Paulo, SBG, p. 297-305.

Bauer, M. \& Largher, G.N. 1958. A preliminary report of the eastern central part of the State of Mato Grosso and a portion of the western part of the state of Goiás, Brasil, Ponta Grossa. DEBSP. Petrobrás. (Relatório técnico interno), 837 p.

Bertini, R.J.; Marshall, L.G.; Gayet, M. \& Brito, P.M. 1993. The vertebrate fauna of the Adamantina and Marília formations, Upper Cretaceous of the Paraná Basin, Southeast Brasil. Neues Jahrbuch fur Mineralogie Geologie und Paleontologie, 188(1):71-101.

Bertini, R.J.; Santucci, R.M.; Ribeiro, L.C.B. \& Arruda-Campos, A.C. 2000. Aeolosaurus (Sauropoda, Titanosauria) from Upper Cretaceous of Brasil. In: JORNADAS ARGENTINAS DE PALEONTOLOGIA DE VERTEBRADOS, 16, 2000. Actas, San Luis, APA. p. 6.

Fernandes, L.A. \& Coimbra, A.M. 1996. A Bacia Bauru (Cretáceo Superior, Brasil). Anais da Academia Brasileira de Ciências, 68(2):195-205.

Fúlfaro, V.J \& Perinotto, J.A.J. 1996. A Bacia Bauru: estado da arte. In: SIMPÓSIO SOBRE O CRETÁCEO DO BRASIL, 4 , Boletim, Águas de São Pedro, UNESP, p. 297-303.

Gibson, S.A.; Thompson, R.N.; Weska, R.K.; Dickin, A.P. \& Leonardos, O.H. 1997. Late Cretaceous rift-related upwelling and melting of the Trindade starting manthe plume head beneath western Brazil. Contributions to Mineralogy and Petrology, 1:303-314.

Kellner, A.W.A. \& Azevedo, S.A.K. 1999. A new sauropod dinosaur (Titanosauria) from the Late Cretaceous of Brasil. In: GONDWANA DINOSAUR SYMPOSIUM, 2, 1999. Proceedings, Tokyo, p. 111-142.

Medeiros, M.A. \& Schultz, C.L. 2000. Dinossauros do Cretáceo médio do Maranhão. In: SIMPÓSIO BRASILEIRO DE PALEONTOLOGIA DE VERTEBRADOS, 2, 2000. Boletim de resumos, Rio de Janeiro, MNRJ, p. 42.

Mezzalira, S. 1989. Os fósseis do Estado de São Paulo. São Paulo, Instituto Geológico, 142 p.

Powell, J.E. 1986. Revisión de los titanosáuridos de América del Sur. Universidad Nacional de Tucumán, Tesis Doctoral, 493 p.

Powell, J.E. 1987. The Late Cretaceous Fauna of Los Alamitos, Patagonia, Argentina. Part IV. The titanosaurids. Revista del Museo Argentino de Ciencias Naturales, 3(3):147-153.

Roxo, M.G.O. 1937. Nota geológica sobre a Chapada dos Guimarães do Mato Grosso. Serviço Geológico e mineralógico, Rio de Janeiro. Notas preliminares e estudos, 15:4-7.

Salgado, L. 2003. Should we abandon the name Titanosauridae? Some comments on the taxonomy of titanosaurians sauropods (Dinosauria). Revista Española de Paleontología 18(1):15-21.

Salgado, L. \& Coria, R.A. 1993. El género Aeolosaurus (Sauropoda, Titanosauridae) en la Formación Allen (CampanianoMaastrichitiano) de la Provincia de Rio Negro, Argentina. Ameghiniana, 30(2):119-128.

Salgado, L.; Coria, R.A. \& Calvo, J.O. 1997a. Evolution of titanosaurid sauropods. I: Phylogenetic analysis based on the postcranial evidence. Ameghiniana, 34:2-32. 
Salgado, L.; Coria, R.A. \& Calvo, J.O. 1997b. Presencia del género Aeolosaurus (Sauropoda, Titanosauridae) en la Formación Los Alamitos, Cretácico Superior de la Provincia de Río Negro, Argentina. Revista Universidade Guarulhos-Geociências 2(6):44-49.

Santucci, R.M. \& Bertini, R.J. 2001. Distribuição paleogeográfica e biocronológica dos titanossauros (Saurischia, Sauropoda) do Grupo Bauru, Cretáceo Superior do Sudeste brasileiro. Revista Brasileira de Geociências, 31(3):307-314.

Santucci, R.M. \& Bertini, R.J. 2002. Distribuição estratigráfica dos titanossauros do Grupo Bauru, Cretáceo Superior continental do sudeste do Brasil. In: SIMPÓSIO SOBRE O CRETÁCEO DO BRASIL, 6, 2002. Boletim, São Pedro, p. 401-408.

Weska, R.K. 1996. Geologia da região diamantífera de Poxoréu e áreas adjacentes, Mato Grosso. São Paulo. Universidade de São Paulo,Tese de Doutoramento, 219 p.
Weska, R.K.; Danni, J.C.M.; Dardene, M.A. \& Perin, A.L. 1988. Contribuição a Estratigrafia do Grupo Bauru da Chapada dos Guimarães, MT. In: CONGRESSO BRASILEIRO DE GEOLOGIA, 35, 1988. Anais, Belém, SBG, 2:905-916.

Weska, R.K.; Svisero, D.P. \& Leonardos, O.H. 1996. Contribuição ao conhecimento do Grupo Bauru no Estado de Mato Grosso, Brasil. In: SIMPÓSIO SOBRE O CRETÁCEO DO BRASIL, 4, 1996. Anais, Rio Claro, p. 289-295.

Zolinger, I. T. 2000. Aspectos mineralógicos e econômicos de diamantes das regiões de Chapada dos Guimarães, Poxoréu, Diamantino, Paranatinga e Alto Paraguai, Mato Grosso. Universidade de São Paulo, Dissertação de Mestrado, 194 p.

Received September, 2003; accepted April, 2004. 